\title{
Immunohistochemical detection of ROS1 is useful for identifying ROS1 rearrangements in lung cancers
}

\author{
Akihiko Yoshida ${ }^{1}$, Koji Tsuta ${ }^{1}$, Susumu Wakai ${ }^{1}$, Yasuhito Arai ${ }^{2}$, Hisao Asamura ${ }^{3}$, \\ Tatsuhiro Shibata ${ }^{2}$, Koh Furuta ${ }^{1}$, Takashi Kohno ${ }^{4}$ and Ryoji Kushima ${ }^{1}$ \\ ${ }^{1}$ Division of Pathology and Clinical Laboratories, National Cancer Center Hospital, Tokyo, Japan; ${ }^{2}$ Division of \\ Cancer Genomics, Center for Medical Genomics, National Cancer Center Research Institute, Tokyo, Japan; \\ ${ }^{3}$ Division of Thoracic Surgery, National Cancer Center Hospital, Tokyo, Japan and ${ }^{4}$ Division of Genome \\ Biology, National Cancer Center Research Institute, Tokyo, Japan
}

\begin{abstract}
The recent discovery and characterization of an oncogenic ROS1 gene fusion in a subset of lung cancers has raised significant clinical interest because small molecule inhibitors may be effective to these tumors. As lung cancers with ROS1 rearrangements comprise only $1-3 \%$ of lung adenocarcinomas, patients with such tumors must be identified to gain optimal benefit from molecular therapy. Recently, immunohistochemical analyses using a novel anti-ROS1 rabbit monoclonal antibody (D4D6) have shown promise for accurate identification of ROS1-rearranged cancers. To validate this finding, we compared the immunostaining results of tissue microarrays (TMAs) containing 17 ROS1-rearranged and 253 ROS1-non-rearranged lung carcinomas. All 17 ROS1-rearranged cancers showed ROS1 immunoreactivity mostly in a diffuse and moderate-to-strong manner with an H-score range of 5-300 (median, 260). In contrast, $69 \%$ of ROS1-non-rearranged cancers lacked detectable immunoreactivity, whereas the remaining $31 \%$ showed reactivity mainly in a weak or focal manner. The H-score for the entire ROS1-non-rearranged group ranged from 0 to 240 (median, 0). The difference in $\mathrm{H}$-score between the two cohorts was statistically significant, and the H-score cutoff $(\geq 150)$ allowed optimal discrimination (94\% sensitivity and $98 \%$ specificity). Similar but slightly less-specific performance was achieved using the extent of diffuse $(\geq 75 \%)$ staining or $\geq 2+$ staining intensity as cutoffs. CD74-ROS1 and EZR-ROS1 fusions were significantly associated with at least focal globular immunoreactivity and plasma membranous accentuation, respectively, and these patterns were specific to ROS1-rearranged cases. Although full-length ROS1 is expressed in some ROS1-non-rearranged cases, we showed that establishment of an optimal set of interpretative criteria makes ROS1 immunohistochemistry a valuable method to rapidly and accurately screen lung cancer patients for appropriate molecular therapy.

Modern Pathology (2014) 27, 711-720; doi:10.1038/modpathol.2013.192; published online 1 November 2013
\end{abstract}

Keywords: adenocarcinoma; immunohistochemistry; lung; ROS1

A significant proportion of lung carcinomas are not amenable to surgical management because they present at advanced stages or recur after primary resection. ${ }^{1}$ Molecular subclassification of tumors is particularly important for such cases because genetic change is the major determinant of the effectiveness of targeted molecular therapy. For example, lung cancers with anaplastic lymphoma kinase $(A L K)$

Correspondence: Dr A Yoshida, MD, PhD, Department of Pathology and Clinical Laboratories, National Cancer Center Hospital, 5-1-1 Tsukiji, Chuo-ku, Tokyo 104-0045, Japan.

E-mail: akyoshid@ncc.go.jp

Received 5 July 2013; accepted 2 September 2013; published online 1 November 2013 gene rearrangements are susceptible to treatment with ALK inhibitors (for example, crizotinib), ${ }^{2}$ and those with a mutation in the gene encoding epidermal growth factor receptor (EGFR) respond to EGFR inhibitors (for example, erlotinib and gefitinib). ${ }^{3}$ The recent discovery and characterization of oncogenic ROS1 gene fusion in lung adenocarcinomas $^{4-8}$ have expanded the list of the molecular subsets of lung cancers. ROS1 encodes a protein tyrosine kinase that belongs to the insulin receptor family. ROS1 is fused to one of a number of genes in lung cancers, including CD74, SLC34A2, EZR, LRIG3, SDC4, TPM3, FIG (also known as GOPC), CCDC6, and KDELR2. ${ }^{4-7,9-12}$ In these fusions, the $3^{\prime}$ region of ROS1 encoding its kinase 
domain is fused to the $5^{\prime}$ region of the respective partner gene. The fusion encodes a chimeric protein with constitutive kinase activity that initiates oncogenic intracellular signal transduction cascades. ${ }^{7,13}$ Preclinical data suggest that ROS1-rearranged cancers respond to ALK inhibitors, ,5,6,9 and a recent clinical trial ${ }^{14}$ revealed a marked inhibition of this molecular subclass by crizotinib. These data underscore the clinical importance of identifying ROS1-rearranged cancers to customize treatment.

As ROS1-rearranged lung cancer comprises only $1-3 \%$ of lung adenocarcinomas, ${ }^{4-9,15}$ the appropriate patients must be selected who will benefit most from molecular therapy. Although these cancers are diagnosed using the reverse transcriptase-polymerase chain reaction (RT-PCR) and/or fluorescence in situ hybridization (FISH), molecular assays are time-consuming, costly, and not suitable for rapid screening. Unfortunately, clinicopathologic features serve poorly for this purpose. Although ROS1-rearranged cancer tends to occur in young non-smokers, ${ }^{5,7,8}$ clinical parameters are not sufficiently predictive for successful triage. Similarly, although the characteristic histological features have been described for this subset, ${ }^{7,8}$ their role is likely limited in the care of patients who present at advanced stages where molecular therapy is most needed because such features are present in only a subset of fusionpositive cases typically as a focal manner. ${ }^{8}$ Recently, Rimkunas et $a l^{9}$ developed a novel anti-ROS1 rabbit monoclonal antibody (D4D6) and proposed the utility of immunohistochemistry for identifying ROS1-rearranged cancers by showing its $100 \%$ (8/8) sensitivity and $100 \%(138 / 138)$ specificity when compared with break-apart FISH. However, the issue is still controversial because other investigators $^{16,17}$ observed ROS1 expression in a significant proportion (20-30\%) of lung carcinomas likely unassociated with gene rearrangement. In this study, we applied this D4D6 antibody to a large number of lung cancers with a known ROS1 rearrangement status to test the utility of immunohistochemistry for molecular subtyping.

\section{Materials and methods}

\section{Case Selection}

After receiving approval from the institutional review board at the National Cancer Center in Tokyo, we constructed TMAs containing 346 primary lung adenocarcinomas by using a tissuearraying instrument (Azumaya, Tokyo, Japan). The tumors were collected from surgical resections with curative intent performed at the National Cancer Center Hospital from 1997 to 2009, and they were enriched for EGFR wild-type cases by using highresolution melting analysis ${ }^{18}(27 \%$ were $E G F R$ mutants). Each tumor was sampled by collecting 2.0-mm-diameter cores from two different representative sites. The TMAs were analyzed by using ROS1 break-apart FISH as described below. After exclusion of 84 cases that either failed to hybridize or lacked an adequate amount of evaluable tumor tissue in the cores, 9 ROS1-rearranged cases and 253 ROS1-non-rearranged cases were identified. The ranges of rearrangement-positive cell rate in the ROS1-rearranged and ROS1-non-rearranged cohorts were $42-84 \%$ and $0-8 \%$, respectively; no case showed borderline $10-20 \%$ range of rearrangement signals. To expand the rearrangement-positive cohort, eight ROS1-rearranged tumors (seven adenocarcinomas and one adenosquamous carcinoma) that were separately identified using RT-PCR were also included, and they were similarly assembled in a TMA as duplicate $2.0-\mathrm{mm}$ cores, except for one case with a limited amount of tissue. Of the 17 ROSrearranged cancers included in this study, 15 were previously reported with their detailed clinicopathologic findings. ${ }^{8}$

\section{FISH}

FISH assays were performed using a custom ROS1 break-apart probe set (Chromosome Science Labo Inc., Sapporo, Japan), which hybridizes with the neighboring $5^{\prime}$ telomeric (RP11-48A22, labeled with SpectrumGreen) and $3^{\prime}$ centromeric (RP11-1036C2, labeled with SpectrumOrange) sequence of the ROS1 gene. This probe set is designed to detect all known ROS1 fusions, including FIG-ROS1, which is unlikely to be detected using a previously described design ${ }^{5,8}$ in which the $5^{\prime}$ probe hybridizes with the RP11-835I21 region. The present probe was internally validated to identify the FIG-ROS1 fusion in the U-118 MG glioblastoma cell line. ${ }^{19}$ FISH images were captured using the Metafer Slide Scanning Platform (MetaSystems, Altlussheim, Germany) to facilitate analysis. Fifty non-overlapping tumor cells with at least one each of $5^{\prime}$ and $3^{\prime}$ signals were examined for each case. The rearrangement-positive cells were defined as those with split signals or isolated red $\left(3^{\prime}\right)$ signals. The specimen was considered as ROS1-rearranged if the rearrangement-positive cells constituted $\geq 15 \%$ of the enumerated tumor cells. This $15 \%$ cutoff value was previously established to accurately differentiate between ROS1-rearranged and ROS1-nonrearranged cases based on RT-PCR data. ${ }^{8}$

\section{Multiplex RT-PCR}

Multiplex RT-PCR was performed as described previously ${ }^{8}$ and was designed to detect the fusion transcripts as follows: CD74-ROS1, EZR-ROS1, SLC34A2-ROS1, FIG-ROS1, LRIG3-ROS1, SDC4ROS1, and TPM3-ROS1. The PCR products were subjected to Sanger sequencing. 


\section{ROS1 Immunohistochemistry}

Immunohistochemical staining was performed on TMA sections, except for one ROS1-rearranged case that was evaluated using the whole section. Fourmicrometer-thick sections were deparaffinized, and heat-induced epitope retrieval was performed with targeted retrieval solution ( $\mathrm{pH}$ 9) (Dako, Carpinteria, CA, USA). The slides were treated with $3 \%$ hydrogen peroxide for $20 \mathrm{~min}$ to block endogenous peroxidase activity. The slides were then incubated with a primary antibody against ROS1 (D4D6, 1:100, Cell Signaling Technology, Danvers, MA, USA) at $4{ }^{\circ} \mathrm{C}$ overnight. Reactivity was detected using the EnVision-FLEX + (Dako). Immunostained slides were scored using the H-score method, which is based on the percentages of cells stained with intensities of $0,1+, 2+$, and $3+$ as follows: $\mathrm{H}$-score $=\sum$ [intensity $(0,1,2,3) \times$ extent of each staining intensity (\%)]. H-scores range from 0 to 300 . Intensity 0 was defined as no detectable staining. Intensity $1+$ was defined as reactivity only detectable at high magnification ( $\times 20-40$ objective). More intense reactivity was divided into moderate $(2+)$ and strong $(3+)$ based on the ease of detection at low magnification ( $\times 4$ objective).

\section{Statistical Analysis}

All data were analyzed using SPSS version 20.0 (IBM Corporation, Somers, NY, USA). The Fisher's exact test and the Mann-Whitney $U$-test were used for categorical and continuous data, respectively. $P$ values were two-tailed, and $P<0.05$ was considered significant.

\section{Results}

\section{Immunohistochemical Analysis of ROS1 Expression}

Immunostaining was evaluated based on the results of two TMA cores for each tumor, except for 12 ROS1-non-rearranged cases for which scoring was performed on one core that contained tumor tissue. All 17 ROS1-rearranged cancers showed ROS1 immunoreactivity primarily in a diffuse and moderate-to-strong manner with an $\mathrm{H}$-score range of 5-300 (median, 260, Figure 1a). In contrast, most (69\%) ROS1-non-rearranged cancers lacked detectable immunoreactivity (Figure 1b), whereas the remaining $31 \%$ showed some degree of reactivity, mostly in a weak or focal manner (Figure 1c). The H-score for the entire ROS1-non-rearranged group ranged from 0 to 240 (median, 0). The difference in H-score between the two cohorts was statistically significant $(P<0.001)$. The staining pattern in all the 95 immunopositive cases (17 ROS1-rearranged and 78 ROS1-non-rearranged cases) was cytoplasmic. The background lung parenchyma included in the TMA cores occasionally showed ROS1 staining in macro- phages (14 cases) and in reactive type II pneumocytes (15 cases, Figure 1d).

\section{Establishment of Immunostaining Interpretative Criteria to Predict Gene Rearrangement}

The distribution of H-scores is illustrated in Figure 2. As the scores were continuous rather than sharply separated into two categories, we attempted to establish an optimal set of criteria that helps to predict ROS1 rearrangement. As there is no universally accepted $\mathrm{H}$-score as a cutoff in the literature, we set a range of $\mathrm{H}$-scores $(0,5,10,20,50,100$, 150,200 , and 250) as the cutoff and calculated test sensitivity and specificity for each condition. This analysis showed that an H-score of $\geq 150$ best discriminated between ROS1-rearranged and -nonrearranged cases with $94 \%$ sensitivity and $98 \%$ specificity (Table 1). Moreover, we set an array of more conventional criteria based on staining extent or intensity and similarly calculated test sensitivity and specificity for each condition. The best separation was achieved when immunopositivity was defined as $\geq 75 \%$ tumor cells labeling with any intensity, and it produced 94\% sensitivity and 90\% specificity (Table 1). Similar results (94\% sensitivity and $87 \%$ specificity) were obtained when the immunopositivity was defined as $\geq 2+$ intensity in any extent.

\section{Correlation of ROS1 Fusion Partner With Staining Pattern}

Among 17 rearrangement-positive cases, data on ROS1 fusion partners were available for 15 cases as follows: CD74-ROS1 (C6;R34), $n=10 ;$ EZR-ROS1 (E10;R34), $n=4$; SLC34A2-ROS1 (S13del2046;R34), $n=1$. Among 10 CD74-ROS1-positive tumors, six showed at least focal globular immunoreactivity, comprising 1-6 round to ovoid intense intracytoplasmic signals measuring $3-8 \mu \mathrm{m}$ in diameter. These globules appeared randomly distributed within the cytoplasm rather than restricted to the perinuclear zones. They occurred within the background of weaker cytoplasmic staining and were occasionally associated with adjacent fine granularity. This pattern was observed in almost all cells in one case (Figure 3a), whereas it was observed in a subset of cells in the remaining five cases (Figure 3b). This pattern was not observed in the remaining four CD74-ROS1-positive tumors and five ROS1-rearranged tumors with partners other than CD74. The association between a globular pattern and $C D 74$ as a fusion partner was statistically significant $(P=0.044)$. One tumor $(P 16)$ that was not subjected to RT-PCR also showed this globular pattern.

Among the four EZR-ROS1-positive tumors, three showed at least focal plasma membranous linear accentuation with occasional fine granular quality. 

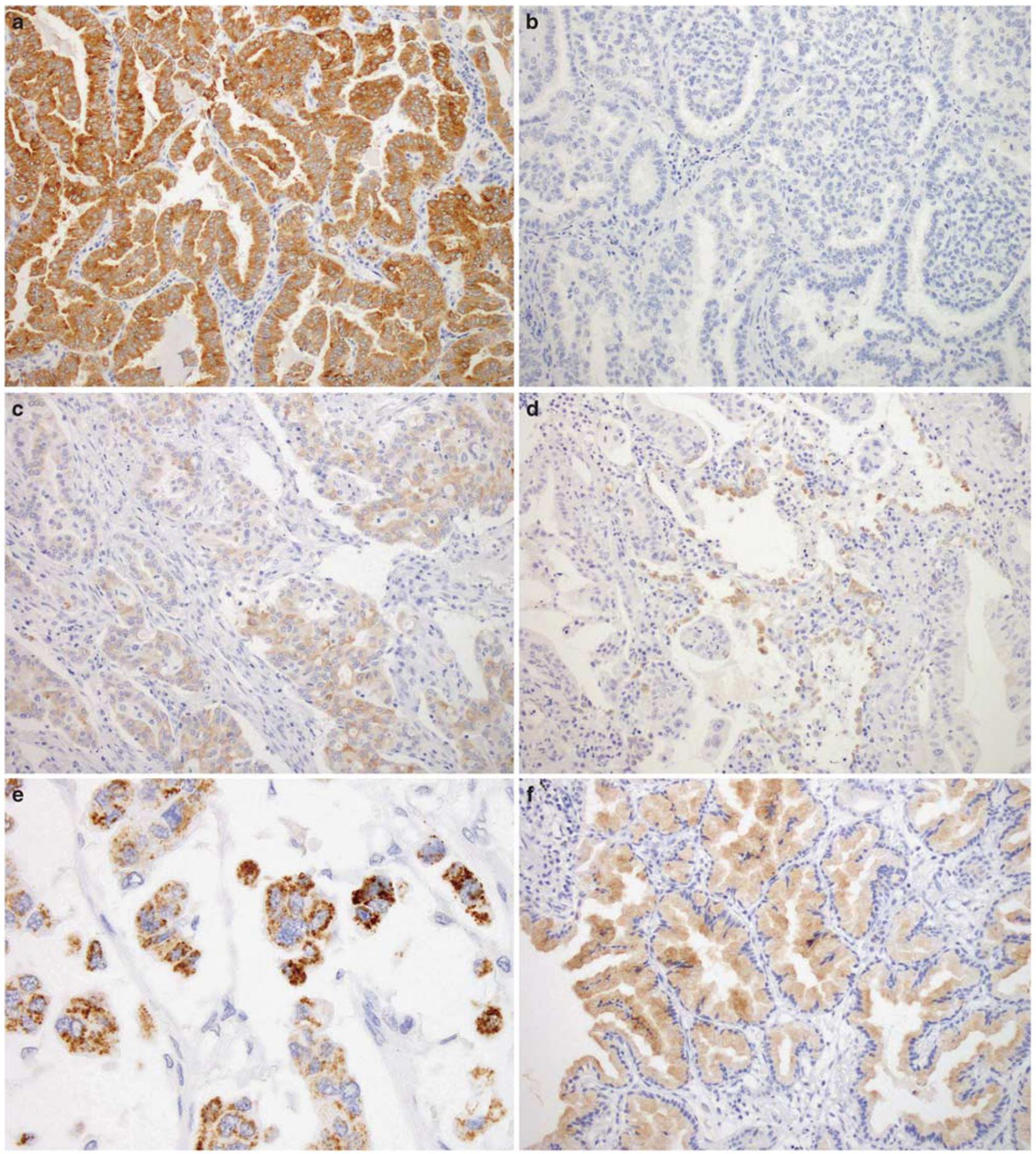

Figure 1 Most ROS1-rearranged cancers showed diffuse and moderate-to-strong ROS1 immunoreactivity (a), whereas 69\% of ROS1-nonrearranged cancers lacked detectable ROS1 expression (b). The remaining 31\% of ROS1-non-rearranged cancers expressed ROS1, mainly in a weak or focal manner (c). Adjacent lung parenchyma showed occasional ROS1 expression in reactive type II pneumocytes (d). The pattern of ROS1 reactivity in some ROS1-non-rearranged tumors was distinctly granular (e). The majority of invasive mucinous adenocarcinomas showed ROS1 reactivity despite the lack of a gene rearrangement (f).

Memebranous accentuation appeared on the lateral surface of tumor cells in two cases (Figure 3c) and along the apical surface in one case (Figure 3d). This pattern was not observed in the remaining EZRROS1-positive tumor and 11 ROS1-rearranged tu- mors with partners other than EZR. The association between membranous accentuation and EZR as a fusion partner was statistically significant $(P=0.009)$. None of the 78 rearrangement-negative tumors with ROS1 expression showed globular 


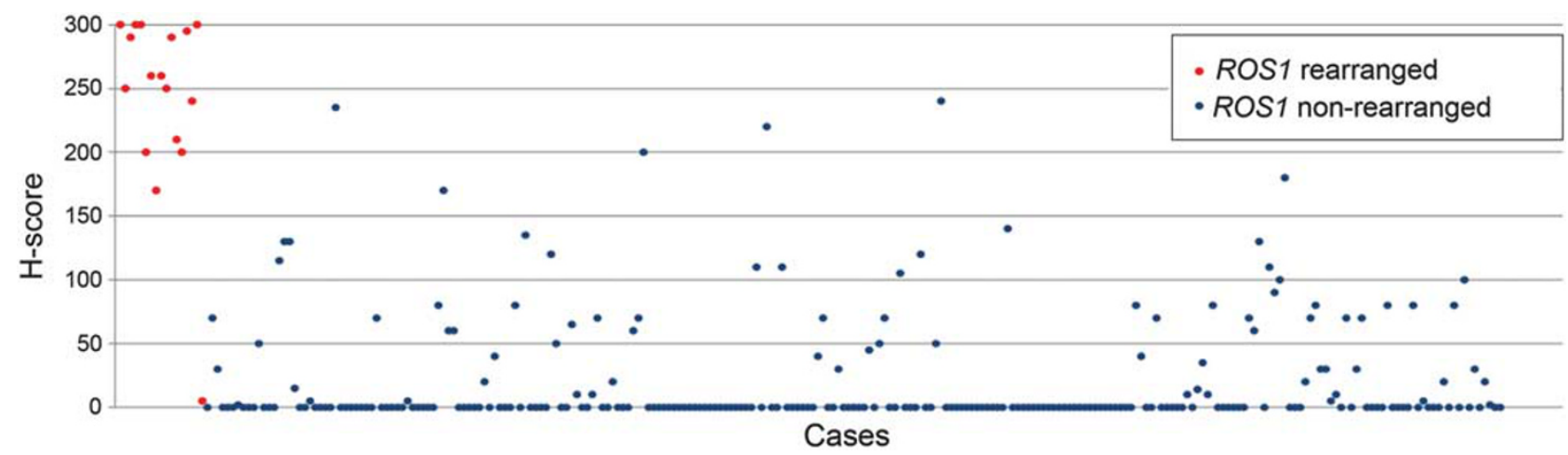

Figure 2 Distribution of H-scores for lung adenocarcinomas determined by using ROS1 immunohistochemistry. Red dots represent scores of ROS1-rearranged cases, and blue dots represent scores of ROS1-non-rearranged cases.

Table 1 Performance of ROS1 immunohistochemical analysis to predict ROS1 rearrangement using an array of interpretative criteria

\begin{tabular}{|c|c|c|c|c|}
\hline Criteria & $\begin{array}{l}\text { The } \\
\text { number of } \\
\text { ROS1- } \\
\text { rearranged } \\
\text { cases that } \\
\text { meet the } \\
\text { criteria } \\
\text { (total } \\
\mathrm{N}=17 \text { ) }\end{array}$ & $\begin{array}{l}\text { The } \\
\text { number of } \\
\text { ROS1-non- } \\
\text { rearranged } \\
\text { cases that } \\
\text { meet the } \\
\text { criteria } \\
\text { (total } \\
\mathrm{N}=253 \text { ) }\end{array}$ & Sensitivity & Specificity \\
\hline \multicolumn{5}{|l|}{ H-score } \\
\hline H-score >0 & 17 & 78 & $100 \%$ & $69 \%$ \\
\hline H-score $\geq 5$ & 17 & 76 & $100 \%$ & $70 \%$ \\
\hline H-score $\geq 10$ & 16 & 72 & $94 \%$ & $72 \%$ \\
\hline H-score $\geq 20$ & 16 & 65 & $94 \%$ & $74 \%$ \\
\hline H-score $\geq 50$ & 16 & 49 & $94 \%$ & $81 \%$ \\
\hline H-score $\geq 100$ & 16 & 20 & $94 \%$ & $92 \%$ \\
\hline H-score $\geq 150^{\mathrm{a}}$ & 16 & 6 & $94 \%$ & $98 \%$ \\
\hline H-score $\geq 200$ & 15 & 4 & $88 \%$ & $98 \%$ \\
\hline H-score $\geq 250$ & 12 & 0 & $71 \%$ & $100 \%$ \\
\hline \multicolumn{5}{|l|}{ Extent } \\
\hline Extent $\geq 1 \%$ & 17 & 78 & $100 \%$ & $69 \%$ \\
\hline Extent $\geq 5 \%$ & 17 & 76 & $100 \%$ & $70 \%$ \\
\hline Extent $\geq 10 \%$ & 16 & 70 & $94 \%$ & $72 \%$ \\
\hline Extent $\geq 50 \%$ & 16 & 48 & $94 \%$ & $81 \%$ \\
\hline Extent $\geq 75 \%^{\mathrm{a}}$ & 16 & 25 & $94 \%$ & $90 \%$ \\
\hline Extent $=100 \%$ & 15 & 6 & $88 \%$ & $98 \%$ \\
\hline \multicolumn{5}{|l|}{ Intensity } \\
\hline Intensity $\geq 2+^{a}$ & 16 & 33 & $94 \%$ & $87 \%$ \\
\hline Intensity $=3+$ & 13 & 8 & $76 \%$ & $97 \%$ \\
\hline
\end{tabular}

${ }^{\mathrm{a}}$ Indicates optimal criteria to predict ROS1 rearrangement.

reactivity or plasma membranous accentuation. The only SLC34A2-ROS1-positive case showed solid cytoplasmic ROS1 staining without distinctive features.

\section{Analysis of Immunopositive ROS1-Non-Rearranged Cases}

Among the 78 immunopositive ROS1-non-rearranged cases, $16(21 \%)$ showed at least focal granular-staining quality (Figure 1e), and the remaining 62 cases $(79 \%)$ showed non-granular solid staining. Twelve tumors (15\%) were morphologically classified as invasive mucinous adenocarcinoma (formerly mucinous bronchioloalveolar carcinoma with invasion; ${ }^{20}$ Figure 1f). They comprised $80 \%$ of the 15 invasive mucinous adenocarcinomas included here. Among the remaining 238 non-mucinous ROS1-non-rearranged cases, we did not observe a clear correlation between histology and immunoreactivity.

\section{Analysis of ROS1-Rearranged Cases with Low Immunostaining}

Two ROS1-rearranged tumors exhibited less ROS1 staining than did the other 15 cases. One (P8) was an adenocarcinoma that was almost purely composed of signet-ring cells (Figure 4a) whose ROS1 rearrangement (EZR-ROS) was confirmed using FISH and RT-PCR. The tumor showed diffuse but weak to moderate ROS1 reactivity with an H-score of 170 (Figure 4b). The other outlier case (P17) was an adenocarcinoma resected from a non-smoking Japanese woman in her 50's. It was positive for FISH with $78 \%$ of tumor cells having rearrangement patterns mostly in the form of isolated $3^{\prime}$ signals (Figure 5a). FISH positivity was confirmed by examining multiple microscopic fields and by using a probe set of different design (RP11-1036C2 for the $3^{\prime}$ probe and RP11-835I21 for the $5^{\prime}$ probe). However, this case showed only weak focal staining with an $\mathrm{H}$-score of 5 (Figure $5 \mathrm{~b}$ ), and this modest reactivity was confirmed using the whole section. Interestingly, multiplex RT-PCR using fresh frozen material did not detect an ROS1 fusion transcript. Further, this case harbored a deletion of EGFR exon 19 and showed diffuse strong immunoreactivity, detected by using an EGFR deletion (E746-A750del)-specific antibody (clone 6B6, 1:100, Cell Signaling Technology) (Figure 5c). As the disease was in the early stage, the patient was successfully treated by surgical resection and did not undergo moleculartargeted therapy. 

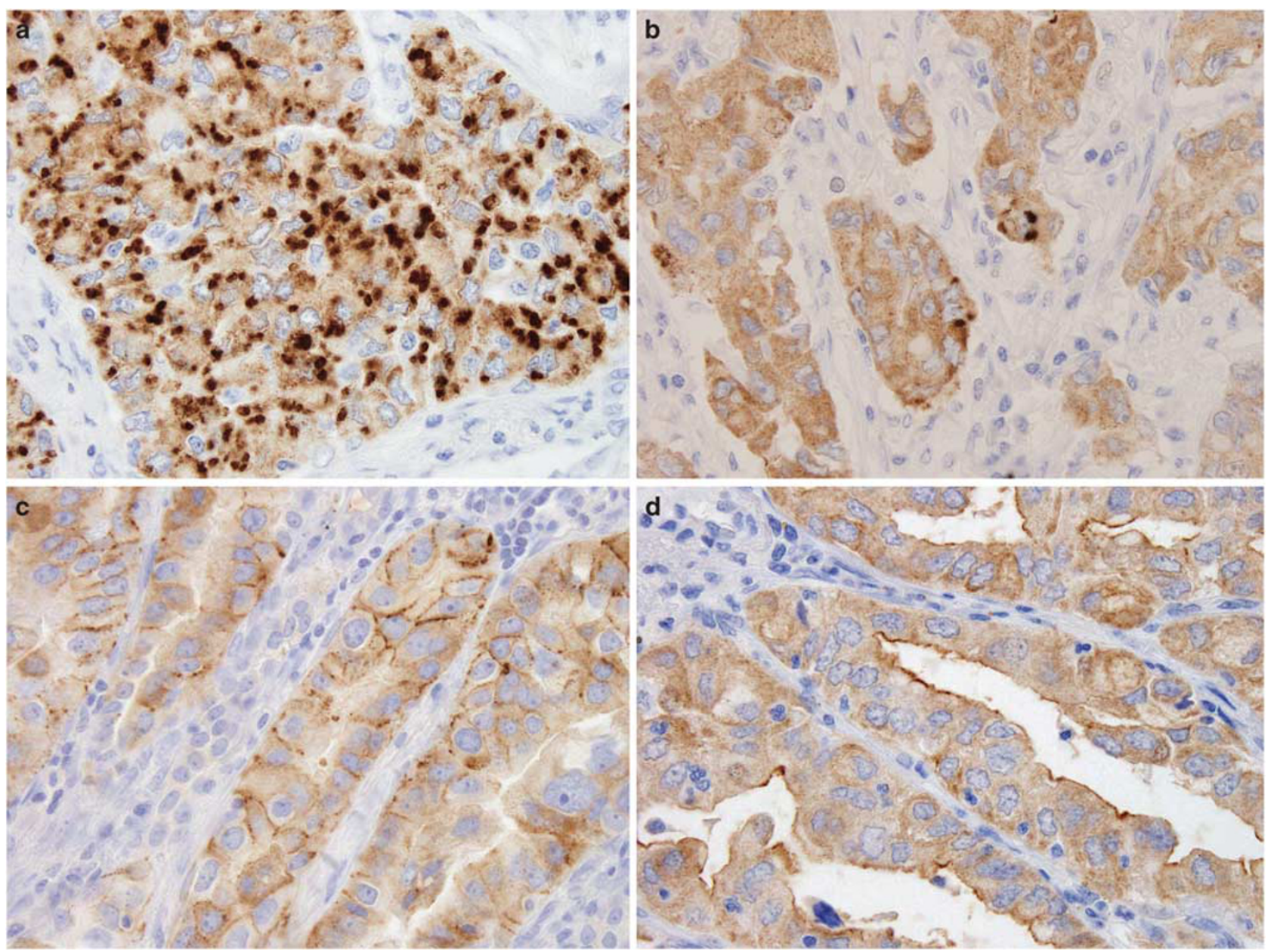

Figure 3 The ROS1 fusion partner correlated with the ROS1-staining pattern. Diffuse (a) or focal (b) intracytoplasmic globular reactivity was observed in 6 of 10 CD74-ROS1-positive cancers. Plasma membranous accentuation with a fine granular quality was observed in 3 of 4 EZR-ROS1-positive tumors; reactivity localizes to the lateral surface in two cases (c) and along the apical surface in one case (d).

\section{Discussion}

We showed here that ROS1 immunoreactivity significantly differed between ROS1-rearranged and non-rearranged lung adenocarcinoma cohorts. However, unlike the observation by Rimkunas et $a l,{ }^{9}$ the reactivity in our present study did not separate the cases into two discrete categories that were in perfect concordance with rearrangement status. In contrast, it produced continuous scores that required statistical treatment for practical application. The reason for this discrepancy may be attributed to the technical differences and the difference in the size of the cases. Our finding of ROS1 expression in $31 \%$ of ROS1-non-rearranged tumors agrees with those of others. For example, in microarray analyses, ROS1 mRNA level was significantly elevated in $20-30 \%$ of non-small cell lung cancers, ${ }^{16}$ and one study ${ }^{15}$ specifically documented the ROS1 mRNA expression independent of gene rearrangement. Similarly, immunohistochemical analyses by Lee et $a l^{17}$ found that $22 \%$ of non-small cell lung carcinomas expressed ROS1.
Taken together, these data highlight the importance of establishing the optimal immunostaining interpretative criteria to predict gene rearrangement.

In our search for such criteria, we found that an $\mathrm{H}$-score of 150 was a reasonable cutoff because of its $94 \%$ sensitivity and 98\% specificity. However, $\mathrm{H}$-score-based criteria may not be practical because $\mathrm{H}$-scores are not routinely used in diagnosis. We therefore tested more conventional sets of criteria that are readily applicable to practice and achieved an optimal test performance ( $94 \%$ sensitivity and $90 \%$ specificity) by using diffuse ( $\geq 75 \%)$ staining of any intensity to define a positive result. Although we noted similar performance using $\geq 2+$ staining intensity, intensity is relatively subjective and is likely more dependent on the staining protocol. In this regard, a previous study ${ }^{9}$ showed $1+$ staining intensity in one-third of the ROS1-rearranged tumors tested, although it did not document the extent of reactivity. ${ }^{9}$ The use of diffuse staining as a criterion to indicate gene rearrangement is reasonable because ROS1 rearrangement is diffusely present within a tumor, ${ }^{8}$ as is typical of early driver 

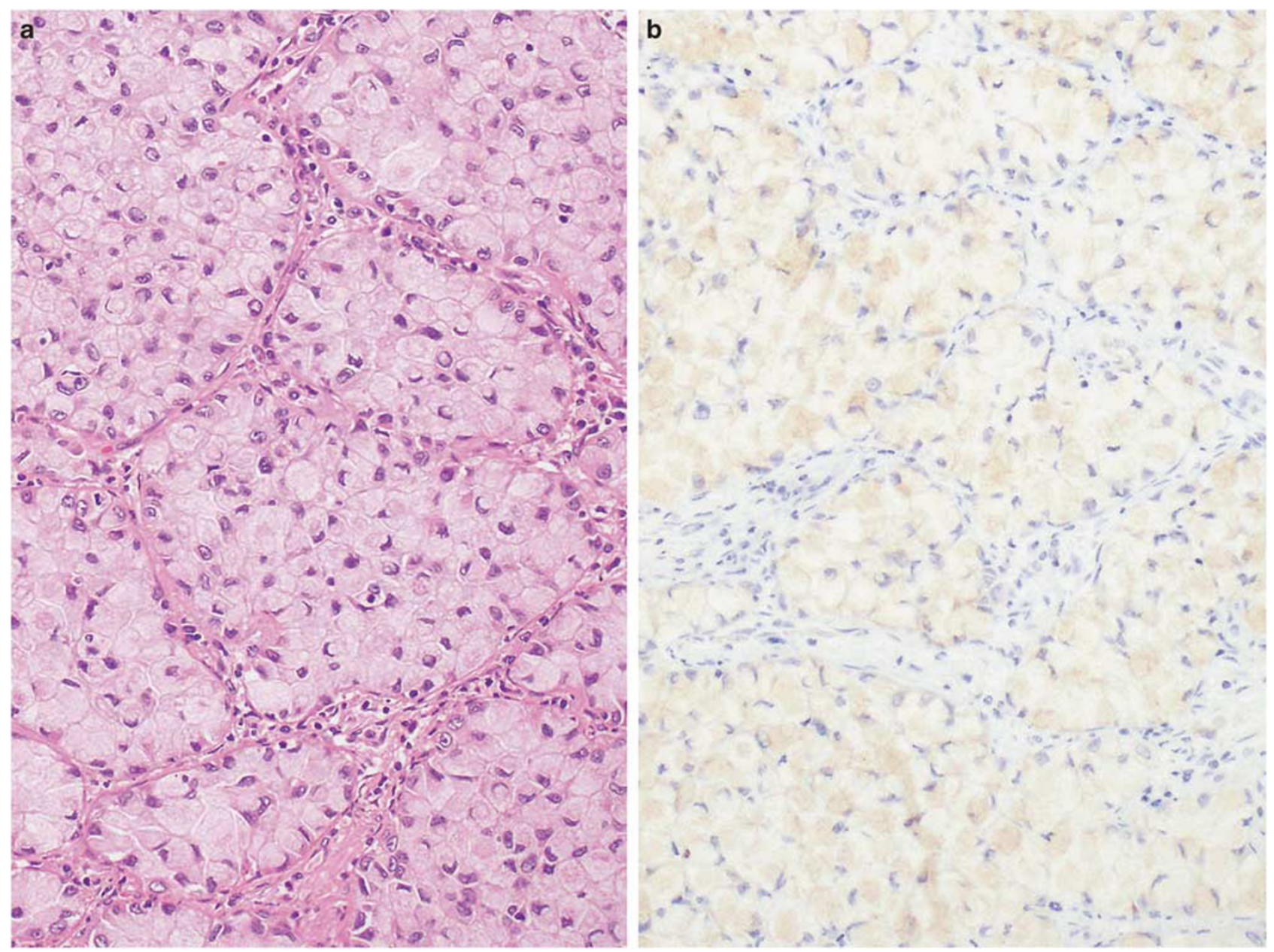

Figure 4 One EZR-ROS1-positive signet-ring cell carcinoma (a) showed diffuse but only weak-moderate ROS1 immunoreactivity (b).

genetic changes such as $A L K$ rearrangement ${ }^{21}$ and EGFR mutation. ${ }^{22}$

We noted a correlation between ROS1 fusion partner genes and the staining patterns, and the result requires validation using a larger cohort. CD74-ROS1 was significantly associated with at least focal globular immunoreactivity. This pattern probably corresponds to the intracytoplasmic puncta that Rimkunas et $a l^{9}$ documented in two of the four CD74-ROS1-positive lung cancers. The mechanism that generates this unusual staining pattern is unknown but may be related to the physiological localization of the CD74 protein that chaperones MHC class II through the intracellular membrane system. ${ }^{23}$ Similarly, the plasma membranous accentuation of reactivity associated with EZR-ROS1 may reflect the subcellular distribution of ezrin protein that links the plasma membrane with the actin cytoskeleton. ${ }^{24}$ These characteristic ROS1-staining patterns were not observed in the 78 rearrangement-negative ROS1expressing cancers in our cohort and, thus, they may be viewed as a rearrangement-specific phenomenon that can be useful for screening. However, we caution that their recognition may not be straightforward because these patterns may be observed only in a fraction of tumor cells (Figure $3 \mathrm{~b}$ ) and because some ROS1-non-rearranged tumors may show at least focal granular staining quality that must be distinguished from CD74-associated globular appearance (compare Figures 1e and 3a).

Our detailed histological analysis of immunohistochemically 'false-positive' cases revealed that invasive mucinous adenocarcinomas were overrepresented (Figure 1f). It is currently unknown whether the reactivity of these tumors represents true full-length ROS1 overexpression or a nonspecific technical artifact perhaps associated with abundant mucin. In any event, histologic appearance should help determine the likelihood of ROS1 rearrangement because invasive mucinous adenocarcinomas are typically associated with KRAS mutation ${ }^{20}$ that hardly coexists with ROS1 rearrangement. Only one invasive mucinous adenocarcinoma with ROS1 rearrangement has been reported to our knowledge. ${ }^{5}$

There were 2 ROS1-rearranged tumors that exhibited less staining than the remaining 15 cases. Case P8 was almost purely composed of signet-ring 

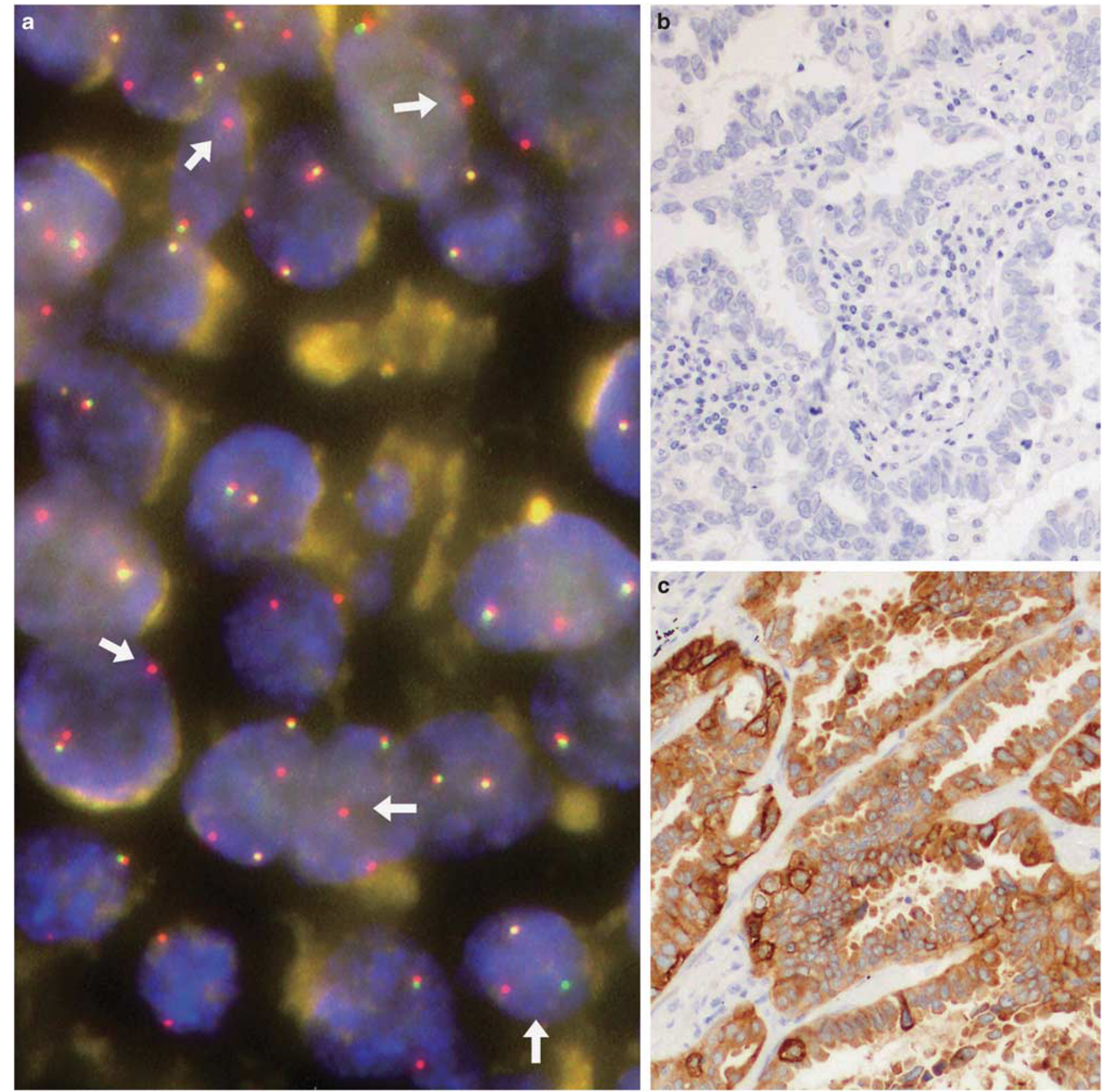

Figure 5 One case showed ROS1 rearrangement determined using FISH (a, arrows indicate rearranged signals), although no ROS1 fusion transcript was amplified using multiplex RT-PCR. ROS1 immunostaining was almost negative (b). The tumor harbored an EGFR exon 19 deletion and diffusely expressed mutant EGFR as detected using immunohistochemistry (c).

cells and reminds us of a reported pitfall of ALK immunohistochemistry for $A L K$-rearranged lung cancers that the staining can be reduced in signetring cells. ${ }^{25}$ Although further study is needed, the potential decrease in immunoreactivity associated with signet-ring cells warrants recognition, particularly because signet-ring cell morphology is characteristic of ROS1-rearranged lung cancer. ${ }^{8}$

The other outlier case (P17) posed a greater challenge to interpret because its driver gene status was not clear. Although FISH analysis indicated gene rearrangement, multiplex RT-PCR did not amplify a ROS1-fusion product. Of note, this was the only case in which a discrepancy occurred between FISH and RT-PCR, of all the cases investigated in the present study as well as in our previous study $^{8}$ on ROS1-rearranged lung cancers. Although one may explain this discordance by hypothesizing a fusion partner that is not covered by the present RT-PCR design, the very low ROS1 immunoreactivity (H-score $=5)$, unlike all other ROS1-rearranged cases, casts doubt on the oncological relevance of ROS1 rearrangement. The presence of an EGFR mutation and diffuse strong overexpression of a 
mutant EGFR in this case further suggest that the tumor is predominantly addicted to the EGFR signaling with only a minor, if any, contribution from ROS1 activity. Only rarely does ROS1 rearrangement coexist with EGFR mutations in lung cancers, and two cases with such a genotype have been reported to show immunohistochemical coexpression of ROS1 and mutant EGFR. ${ }^{9}$ The present case is, instead, reminiscent of two ALKimmunonegative adenocarcinomas reported by Sasaki et $a^{26}$ that harbored an $A L K$-rearrangement (confirmed by FISH) and an EGFR mutation. Future studies such as those using comprehensive sequencing methods may clarify the underlying mechanism that accounts for these unusual disparities. If this case P17 were excluded from the ROS1-rearranged cohort, the sensitivity of ROS1 immunohistochemistry would reach $100 \%$ by using the criteria that we have proposed (that is, H-score $\geq 150$, extent $\geq 75 \%$, or intensity $\geq 2+$ ).

In summary, our present results agree with those reported by Rimkunas et $a l^{9}$ in that ROS1 immunohistochemistry by using a newly developed antibody is useful for screening of lung cancer patients for molecular therapy. However, as fulllength ROS1 is expressed in a proportion of ROS1non-rearranged cases, establishment of optimal interpretative criteria is critical to achieve concordance with genetic status. High H-score ( $\geq 150$ ), diffuse extent, or moderate-to-strong staining intensity provide helpful clues to predict ROS1 rearrangement. Globular reactivity and plasma membranous accentuation correlate with $C D 74$ and $E Z R$ as fusion partners, and these patterns are likely to be fusion-specific. Although ROS1 immunohistochemistry is unlikely to replace confirmatory molecular assays, we expect that it will become an integral part of diagnostic algorithm in thoracic oncology. For example, if ROS1 immunostaining is negative or only focally positive, such a case will be almost certainly negative for ROS1 rearrangement, thus precluding the need of molecular analysis. In contrast, if a diffuse-positive staining is observed, particularly with a moderate-strong intensity, the possibility of ROS1 rearrangement is high and the case should be sent for molecular confirmation. We further suspect that ROS1 immunohistochemistry may find additional utility in wider clinical field in the future because ROS1 rearrangements have also been reported in a growing number of nonpulmonary tumors. ${ }^{19,27,28}$

\section{Acknowledgments}

We thank Sachiko Miura, Chizu Kina, Yuko Adegawa, and Ryosuke Yamaga for their superb technical assistance. This work was supported in part by the Program for Promotion of Fundamental Studies in Health Sciences from the National Institute of Biomedical Innovation (NIBIO), Grants-in-Aid from the Ministry of Health, Labour and Welfare for the 3rd-term Comprehensive 10-year Strategy for Cancer Control, and the National Cancer Center Research and Development Fund. The National Cancer Center Biobank is supported by the National Cancer Center Research and Development Fund, Japan.

\section{Disclosure/conflict of interest}

The authors declare no conflict of interest.

\section{References}

1 Sawabata N, Asamura H, Goya T, et al. Japanese Lung Cancer Registry Study: first prospective enrollment of a large number of surgical and nonsurgical cases in 2002. J Thorac Oncol 2010;5:1369-1375.

2 Kwak EL, Bang YJ, Camidge DR, et al. Anaplastic lymphoma kinase inhibition in non-small-cell lung cancer. N Engl J Med 2010;363:1693-1703.

3 Lynch TJ, Bell DW, Sordella R, et al. Activating mutations in the epidermal growth factor receptor underlying responsiveness of non-small-cell lung cancer to gefitinib. N Engl J Med 2004;350:2129-2139.

4 Rikova K, Guo A, Zeng Q, et al. Global survey of phosphotyrosine signaling identifies oncogenic kinases in lung cancer. Cell 2007;131:1190-1203.

5 Bergethon K, Shaw AT, Ou SH, et al. ROS1 rearrangements define a unique molecular class of lung cancers. J Clin Oncol 2012;30:863-870.

6 Davies KD, Le AT, Theodoro MF, et al. Identifying and targeting ROS1 gene fusions in non-small cell lung cancer. Clin Cancer Res 2012;18:4570-4579.

7 Takeuchi K, Soda M, Togashi Y, et al. RET, ROS1 and ALK fusions in lung cancer. Nat Med 2012;18:378-381.

8 Yoshida A, Kohno T, Tsuta K, et al. ROS1-rearranged lung cancer: a clinicopathologic and molecular study of 15 surgical cases. Am J Surg Pathol 2013;37: $554-562$.

9 Rimkunas VM, Crosby K, Kelly M, et al. Analysis of receptor tyrosine kinase ROS1 positive tumors in nonsmall cell lung cancer: identification of a FIG-ROS1 fusion. Clin Cancer Res 2012;18:4449-4457.

10 Suehara Y, Arcila M, Wang L, et al. Identification of KIF5B-RET and GOPC-ROS1 fusions in lung adenocarcinomas through a comprehensive mRNA-based screen for tyrosine kinase fusions. Clin Cancer Res 2012;18:6599-6608.

11 Govindan R, Ding L, Griffith M, et al. Genomic landscape of non-small cell lung cancer in smokers and never-smokers. Cell 2012;150:1121-1134.

12 Seo JS, Ju YS, Lee WC, et al. The transcriptional landscape and mutational profile of lung adenocarcinoma. Genome Res 2012;22:2109-2119.

13 Arai Y, Totoki Y, Takahashi $\mathrm{H}$, et al. Mouse model for ROS1-rearranged lung cancer. PLoS One 2013;8: e56010.

14 Shaw A, Camidge D, Engelman J, et al. Clinical activity of crizotinib in advanced non-small cell lung cancer (NSCLC) harboring ROS1 gene rearrangement. J Clin Oncol 2012;30:Suppl; abstr 7508.

15 Li C, Fang R, Sun Y, et al. Spectrum of oncogenic driver mutations in lung adenocarcinomas from East Asian never smokers. PLoS One 2011;6:e28204. 
16 Acquaviva J, Wong R, Charest A. The multifaceted roles of the receptor tyrosine kinase ROS in development and cancer. Biochim Biophys Acta 2009;1795: $37-52$.

17 Lee HJ, Seol HS, Kim JY, et al. ROS1 receptor tyrosine kinase, a druggable target, is frequently overexpressed in non-small cell lung carcinomas via genetic and epigenetic mechanisms. Ann Surg Oncol 2013;20: 200-208.

18 Fukui T, Ohe Y, Tsuta K, et al. Prospective study of the accuracy of EGFR mutational analysis by high-resolution melting analysis in small samples obtained from patients with non-small cell lung cancer. Clin Cancer Res 2008;14:4751-4757.

19 Charest A, Lane K, McMahon K, et al. Fusion of FIG to the receptor tyrosine kinase ROS in a glioblastoma with an interstitial del(6)(q21q21). Genes Chromosomes Cancer 2003;37:58-71.

20 Travis WD, Brambilla E, Noguchi M, et al. International Association for the Study of Lung Cancer/ American Thoracic Society/European Respiratory Society International multidisciplinary classification of lung adenocarcinoma. J Thorac Oncol 2011;6: 244-285.

21 Camidge DR, Kono SA, Flacco A, et al. Optimizing the detection of lung cancer patients harboring anaplastic lymphoma kinase (ALK) gene rearrangements potentially suitable for ALK inhibitor treatment. Clin Cancer Res 2010;16:5581-5590.

22 Yatabe Y, Matsuo K, Mitsudomi T. Heterogeneous distribution of EGFR mutations is extremely rare in lung adenocarcinoma. J Clin Oncol 2011;29: 2972-2977.

23 Stumptner-Cuvelette P, Benaroch P. Multiple roles of the invariant chain in MHC class II function. Biochim Biophys Acta 2002;1542:1-13.

24 Bruce B, Khanna G, Ren L, et al. Expression of the cytoskeleton linker protein ezrin in human cancers. Clin Exp Metastasis 2007;24:69-78.

25 Murakami Y, Mitsudomi T, Yatabe Y. A screening method for the ALK fusion gene in NSCLC. Front Oncol 2012;2:24.

26 Sasaki T, Koivunen J, Ogino A, et al. A novel ALK secondary mutation and EGFR signaling cause resistance to ALK kinase inhibitors. Cancer Res 2011;71: 6051-6060.

27 Lee J, Lee SE, Kang SY, et al. Identification of ROS1 rearrangement in gastric adenocarcinoma. Cancer 2013;119:1627-1635.

$28 \mathrm{Gu}$ TL, Deng X, Huang F, et al. Survey of tyrosine kinase signaling reveals ROS kinase fusions in human cholangiocarcinoma. PLoS One 2011;6:e15640. 